\title{
Evaluation of Doppler Velocimetry of Middle Cerebral and Umbilical Arteries in High-Risk Pregnancie
}

Y S Arun Kumar Reddy ${ }^{1} \&$ Shashidhar sangineni $*^{2}$

\section{Article History \\ Received: 30.08 .2020 \\ Accepted: 15.09 .2020 \\ Published: 20.09.2020 \\ Journal homepage: \\ https://www.easpublisher.com/easjrit}

${ }^{1}$ Associate Professor, Department of Radio Diagnosis,

${ }^{2}$ Assistant Professor in Radiodiagnosis, Kamineni Institute of Medical Sciences, Narketpally, Nalgonda, Telangana. India

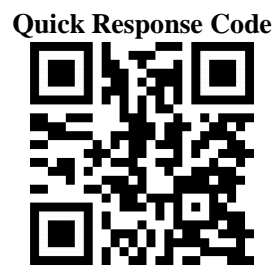

Abstract: Background: Antepartum foetal surveillance is the cornerstone of management aimed at reducing maternal and perinatal mortality and morbidity. Doppler velocimetry of the uteroplacental and fetoplacental circulation can be used to further evaluation in such complications of pregnancy as foetal growth restriction. Aims and Objectives: To study the Systolic / Diastolic ratio (S/D ratio), pulsatility index (PI) and resistance index (RI) of the umbilical artery and middle cerebral arteries in high-risk pregnancies. The ratio of middle cerebral to umbilical artery blood velocity in high-risk pregnancies. Also, to evaluate the role of these blood flow indices in the prediction of adverse foetal outcome. Materials and Methods: This prospective study included 90 pregnant patients with high-risk factors in the III trimester after 30 weeks who got admitted to Department of OBG, Mamata Academy of Medical Sciences, Bachupally, Hyderabad, from October 2018 to November 2019. Results: Total number of 90 high-risk cases were studied. In these 46 cases had intrauterine growth retardation. A ratio of Middle cerebral artery RI / Umbilical artery RI shows more sensitivity and specificity than MCA RI (60\% Vs $28 \%)$ and (75\% Vs 67\%) respectively. It was also more sensitive and specific than Umbilical artery RI (61\% Vs 50\%) and (75\% Vs $\& 72 \%$ ) respectively. However, the most sensitive parameter was Middle Cerebral Artery PI (95\%), and the most specific parameter was Umbilical artery PI (81\%). Conclusion: It has been proved that Doppler technology has a significant impact on our ability to assess the physiological status of the foetus. It can help in evaluating the changes in the foetal circulation. This study suggests that Doppler assessment of fetoplacental circulation, including both middle cerebral artery and the umbilical artery is an excellent prognostic indicator than individual vessels for the prediction of adverse foetal outcomes in high-risk pregnancies. Also, the umbilical artery and middle cerebral artery pulsatility indices are equally an important tool in predicting adverse foetal outcome.

Keywords: Doppler Velocimetry; Umbilical Artery; Middle Cerebral Artery; Systolic/ Diastolic ratio; Pulsatility index; Resistance index; high-risk pregnancies.

Copyright (C) 2020 The Author(s): This is an open-access article distributed under the terms of the Creative Commons Attribution 4.0 International License (CC BY-NC 4.0) which permits unrestricted use, distribution, and reproduction in any medium for non-commercial use provided the original author and source are credited.

\section{INTRODUCION}

Antepartum foetal surveillance is the cornerstone of management targeted at deceasing maternal and perinatal fatality and morbidity. It must be highlighted that applications of ultrasound imaging have revolutionized the antepartum foetal assessment as a whole. Doppler ultrasound is a non-invasive method of evaluating blood flow in the fetoplacental and uteroplacental circulation. This method uses sound waves to measure the flow of blood through a blood vessel (Malik, R., \& Saxena, A. 2013).

The use of this technique for foetal investigations was first reported in 1977 when Fitz Gerald and Drumm demonstrated the Doppler frequency shift waveform from the umbilical arterial circulation. The incorporation of Doppler-derived blood indices and various haemodynamic factors into the standard anatomic ultrasound assessment, this allows the additional evaluation of a variety of physiologic parameters for Obstetrics and Gynaecology specialists (Malik, R., \& Saxena, A. 2013; Ebrashy, A. et al., 2005; \& Cruz-Martínez, R. et al., 2011).

In Obstetrics, Doppler velocimetry of the fetoplacental and uteroplacental circulation can be used to further evaluate the complications of pregnancy as foetal growth restriction and other forms of foetal distress that result from foetal hypoxemia or asphyxia (Cruz-Martínez, R. et al., 2011). Thus, Doppler studies are essential in making the critical distinction between a foetus that is actual growth restricted and one who is small and healthy (Maulik, D. 1989).

Doppler velocimetry is the best method of surveillance for foetal hypoxemia in high-risk 
pregnancy. It is a non-invasive method for assessing foetal circulation. Umbilical and Middle cerebral arteries are routinely assessed. Middle cerebral artery Doppler measurement is a well-known modality for detecting foetal compromise. Studies have shown that MCA Doppler abnormalities were associated with hypoxia and adverse perinatal outcome (Malik, R., \& Saxena, A. 2013; Ebrashy, A. et al., 2005; \& CruzMartínez, R. et al., 2011; Maulik, D. 1989; \& GILES, W. B. et al., 1985).

In high-risk pregnancies like PIH and IUGR, MCA blood flow is increased by reducing the resistance index, i.e., called brain sparing effect. Doppler study gives valuable information about the hemodynamic situation of the foetus and is a practical diagnostic test of foetal jeopardy that helps in the management of highrisk pregnancy (Vyas, S. et al., 1990). Up to 16 weeks of gestation, an early diastolic notch is usually present in the uterine artery. Abnormalities in uterine artery Doppler are the presence of diastolic notch in the second trimester. It is a predictor of pre-eclampsia and IUGR (Burns, P. N. 1987; Lakhkar, B. N. et al., 2006; \& Hernandez-Andrade, E. et al., 2013).

The use of Doppler can be credited, leading to significant decreasing in perinatal morbidity and mortality. The colour Doppler ultrasound gives information directly on vascular resistance and indirectly on blood flow (Bano, S. et al., 2010).

This study has been undertaken to detect the foetal compromise and early termination to improve the perinatal outcome. This study was carried out to evaluate the role of Doppler velocimetry of fetoplacental and uteroplacental circulation in the prediction of abnormal perinatal outcomes, in high-risk pregnancies.

\section{Methodology}

This prospective study included 90 pregnant patients with high-risk factors in the III trimester after 30 weeks who got admitted to the Department of OBG, Mamata Academy of Medical Sciences, Bachupally, Hyderabad, from October 2018 to November 2019.

Patient recruitment: The patients were selected by analysing their well-documented length of pregnancy, singleton pregnancy, and gestational age beyond 30 weeks. Patients with threatened preterm labour and with confusing or wrong dates were excluded from this study.

High-risk factors considered: Pre-eclampsia, Intra Uterine Growth Restriction, Gestational diabetes mellitus, Anaemia, Previous Caesarean section, Bad obstetric history, $\mathrm{Rh}$ isoimmunisation.

Abnormal perinatal outcome: The perinatal outcome was considered abnormal when any one or a combination of the following was present.

1. Perinatal death

2. Thick meconium stained liquor

3. Apgar $<7$ at 5 minutes

4. Respiratory complications within 72 hours of birth

5. NICU admission within 72 hours of birth.

All the patients selected for this study underwent history taking and examination at admission to the ward. The routine antenatal blood and urine investigations were done. Doppler velocity waveforms of umbilical and middle cerebral were taken for both normal and high-risk pregnancies.

A colour Doppler ultrasound apparatus equipped with $3.75 \mathrm{MHz}$ transducers was used for the Doppler study. All the examinations were made with the patient lying in a semi-recumbent position with a lateral tilt. Doppler transducer was placed on the abdominal wall over the uterus and carefully manipulated till Doppler signals appropriate for those particular vessels were identified. All the examinations were performed only during foetal apnoea and foetal inactivity.

The signal was recorded for a minimum of 5 to 8 cycles with blood flow velocity waveforms of similar shape and amplitude and satisfactory quality. Then the image was frozen, and measurements were taken.

Statistical analysis: The collected research data was compiled and analysed with SPSS software.

\section{RESUlTS}

In the present study, out of 90 cases were studied, $47.78 \%$ of cases were between $21-25$ years. The mean age found was $23.73 \pm 4.57$.

Table 1: Tabular representation of Age distribution among different patients

\begin{tabular}{ccc}
\hline Age in years & No. of cases & Percentage (\%) \\
\hline $16-20$ & 27 & 30 \\
$21-25$ & 43 & 47.78 \\
$26-30$ & 13 & 14.44 \\
$31-35$ & 5 & 5.56 \\
$36-40$ & 2 & 2.22 \\
Total & 90 & 100 \\
\hline
\end{tabular}


Out of 90 cases, 53 were mild preeclampsia, of which 15 cases have an adverse outcome (28.33\%). 37 were severe preeclampsia, of which 26 cases with adverse outcome $(71.27 \%)$.

Table 2: Incidence of outcome in relation to severity of preeclampsia

\begin{tabular}{cccc}
\hline Parameters & No. of cases & Adverse outcome & Percentage (\%) \\
\hline Mild preeclampsia & 53 & 15 & 28.33 \\
Severe preeclampsia & 37 & 26 & 71.27 \\
Total & 90 & 41 & 45.55 \\
\hline
\end{tabular}

Abnormal Umbilical PI value is increased in the number of cases, and Umbilical RI value increased in cases which showed adverse perinatal outcome. In our study, umbilical artery PI was more sensitive.

Table 3: Correlation of umbilical artery velocimetry and perinatal outcome

\begin{tabular}{ccccc}
\hline Parameters & Normal outcome & Percentage (\%) & Adverse outcome & Percentage (\%) \\
\hline Normal PI & 54 & 60 & 07 & 7.78 \\
Increased PI & 06 & 6.67 & 23 & 25.56 \\
Normal RI & 49 & 54.44 & 02 & 2.22 \\
Abnormal RI & 15 & 16.67 & 24 & 26.67 \\
Normal S/D ratio & 45 & 50 & 2 & 2.22 \\
Abnormal S/D ratio & 18 & 20 & 25 & 27.78 \\
\hline
\end{tabular}

Middle cerebral artery PI was decreased in cases with $68 \%$ positive predictive value

Table 4: Correlation of MCA PI and adverse perinatal outcome

\begin{tabular}{cccc}
\hline Parameters & Number of Patients & Adverse perinatal outcome & PPV \\
\hline Normal & 47 & 21 & 68 \\
MCA PI $>1$ & & & \\
Abnormal & 18 & 04 & 22 \\
MCA PI $<1$ & & & \\
\hline
\end{tabular}

Table 5: Comparative Analysis of results

\begin{tabular}{ccccc}
\hline Parameters & Sensitivity (\%) & Specificity (\%) & PPV (\%) & NPV (\%) \\
\hline Um PI & 79 & 97.33 & 94.56 & 93.11 \\
Um RI & 96.33 & 92.54 & 64.33 & 98.69 \\
MCA PI & 81.73 & 90.87 & 81.77 & 92.33 \\
\hline
\end{tabular}

\section{Discussion}

Doppler ultrasonography is extensively used in the examination of umbilical arteries for evaluation of uteroplacental circulation and predicting adverse perinatal outcomes (Maged, A. M. et al., 2014), recent studies have proposed the importance of MCA Doppler assessment for antenatal monitoring (Burns, P. N. 1987; Lakhkar, B. N. et al., 2006; Hernandez-Andrade, E. et al., 2013; \& Maged, A. M. et al., 2014).

The objective of foetal Doppler is to detect any hemodynamic changes at the earliest and to assess the placental dysfunction and the consequences of this on foetal growth and well-being. In our study, the umbilical artery S/D ratio is abnormal in 96 cases. Low birth weight babies are 76 and 3 were NND due to extreme prematurity (Devore, G.R. 2015).

This study shows that the Doppler study in the third trimester is instrumental in predicting the foetal outcome in high-risk pregnancy. Abnormal MCA/UA Doppler ratio is strongly correlated with worse foetal prognosis. Foetuses with abnormal Doppler velocimetry had a significantly higher incidence of preterm birth, low birth weight, and admission to NICU. Late-onset SGA foetuses with normal Doppler velocimetry on diagnosis show the progression from 37 weeks gestation with worsening uteroplacental ratio followed by a decrease in MCA pulsating index(PI) (Seal, S. L. et al., 2012; \& Long, P. A., \& Oats, J. N. 1987).

There were a more significant number of caesarean section and NICU admission in abnormal Doppler indices in various studies (Andersch, B. et al., 1984). Repetitive Doppler monitoring in pregnancy has been a great help to improve perinatal outcome in complicated pregnancies. The Doppler patterns follow a longitudinal trend with new changes in the umbilical artery, followed by the middle cerebral artery.

The venous change follows the arterial pattern and occurs in the severely compromised foetus and predicts the poor perinatal outcome. Abnormal Doppler with a nonreactive NST is definitely associated with an adverse perinatal outcome need immediate delivery. In our study, the caesarean section rate is more in cases with abnormal Doppler. In patients with umbilical artery S/D ratio alone, vaginal delivery occurred in $61 \%$ of cases. But in a patient with absent end-diastolic 
flow. $73 \%$ cases ended in caesarean section (Bhatt, C. J. et al., 2003; Hazra, S. K. et al., 2013; \& Gramellini, D. et al., 1992).

Abnormal MCA/PI Doppler ratio correlates with foetal prognosis. In present study ratio of PI of Middle cerebral artery/ umbilical artery shows $78 \%$ positive predictive value. According to the earlier reported study, assessment of MCA/UA PI index provide information in predicting perinatal outcome when compared with umbilical or middle cerebral artery Doppler indices alone (Gramellini, D. et al., 1992; Arduini, D., \& Rizzo, G. 1992; \& Kassanos, D. et al., 2003).

In the present study, out of 90 cases were studied, 25 showed adverse perinatal outcome. 6were perinatal death and 13 had the low Apgar score at 5minutes and six were admitted to NICU.

To improve the neonatal outcome, we need to do the caesarean section in cases with abnormal Doppler. Good NICU care is required for such babies. Close monitoring with NST and repeated Doppler studies are beneficial in such a case.

\section{CONCLUSION}

This study was carried out to investigate the role of Doppler velocimetry of fetoplacental circulation in the prediction of abnormal perinatal outcomes, in high-risk pregnancies. In all high-risk pregnancies, umbilical artery S/D ratio was more sensitive and had got more negative predictive value than umbilical artery and MCA pulsatility index and the ratio of the middle cerebral artery (MCA) and umbilical artery (UA) resistance indices (MCA/UA) in predicting adverse foetal outcome.

In all high-risk pregnancies, the middle cerebral artery pulsatility index was more specific and has got more positive predictive value than umbilical artery S/D ratio and uterine artery resistance index in predicting adverse foetal outcome. The MCA Pulsatility index was more accurate than each of its components in the diagnosis of foetal morbidity and compromise.

The decreased sensitivity and negative predictive value of the ratio of the middle cerebral artery (MCA) and umbilical artery (UA) resistance indices are due to the increased number of falsenegative values when compared to umbilical artery S/D ratio. This is clearly explained by the fact that in extreme cases of IUGR, instead of decreased middle cerebral artery resistance, there will be normal or increased resistance due to an increase in intracranial tension because of brain oedema.

\section{REFERENCES}

1. Andersch, B., Svensson, A., \& Hansson, L. (1984). Characteristics of Hypertension in Pregnancy: A retrospective study of 261 consecutive cases. Acta Obstetricia et

Gynecologica Scandinavica, 63(sup118), 33-38.

2. Arduini, D., \& Rizzo, G. (1992). Prediction of fetal outcome in small for gestational age fetuses: comparison of Doppler measurements obtained from different fetal vessels. Journal of Perinatal Medicine-Official Journal of the WAPM, 20(1), 2938.

3. Bano, S., Chaudhary, V., Pande, S., Mehta, V. L., \& Sharma, A. K. (2010). Color doppler evaluation of cerebral-umbilical pulsatility ratio and its usefulness in the diagnosis of intrauterine growth retardation and prediction of adverse perinatal outcome. The Indian journal of radiology \& imaging, 20(1), 20.

4. Bhatt, C. J., Arora, J., \& Shah, M. S. (2003). Role of color doppler in pregnancy induced hypertension (a study of 100 cases). Indian journal of Radiology and Imaging, 13(4), 417.

5. Burns, P. N. (1987). Doppler flow estimations in the fetal and maternal circulations: principles, techniques and some limitations. In Doppler ultrasound measurements of maternal fetal haemodynamics (pp. 43-76). Perinatology Press, Ithaca, NY.

6. Cruz-Martínez, R., Figueras, F., HernandezAndrade, E., Oros, D., \& Gratacos, E. (2011). Fetal brain Doppler to predict cesarean delivery for nonreassuring fetal status in term small-forgestational-age fetuses. Obstetrics \& Gynecology, 117(3), 618-626.

7. Devore, G.R. (2015). The importance of the uteroplacental ratio in the evaluation of fetal wellbeing in S.GA and AGA fetuses. Am J Obstet Gyeaenacol. 213(1), 5-15.

8. Ebrashy, A., Azmy, O., Ibrahim, M., Waly, M., \& Edris, A. (2005). Middle cerebral/umbilical artery resistance index ratio as sensitive parameter for fetal well-being and neonatal outcome in patients with preeclampsia: case-control study. Croatian medical journal, 46(5) (5), 821-825.

9. GILES, W. B., TRUDINGER, B. J., \& BAIRD, P. J. (1985). Fetal umbilical artery flow velocity waveforms and placental resistance: pathological correlation. BJOG: An International Journal of Obstetrics \& Gynaecology, 92(1), 31-38.

10. Gramellini, D., Folli, M. C., Raboni, S., Vadora, E., \& Merialdi, A. (1992). Cerebral-umbilical Doppler ratio as a predictor of adverse perinatal outcome. Obstetrics and gynecology,79(3), 416420 .

11. Hazra, S. K., Dash, K. K., Chaudhuri, A., Ghosh, M. K., Banerjee, D., \& Guha, S. (2013). A prospective study of doppler velocimetry in pregnancy-induced hypertension in a rural population of a developing country. Journal of 
Basic and Clinical Reproductive Sciences, 2(2), 127-131.

12. Hernandez-Andrade, E., Stampalija, T., \& Figueras, F. (2013). Cerebral blood flow studies in the diagnosis and management of intrauterine growth restriction. Current Opinion in Obstetrics and Gynecology, 25(2), 138-144.

13. Kassanos, D., Siristatidis, C., Vitoratos, N., Salamalekis, E., \& Creatsas, G. (2003). The clinical significance of Doppler findings in fetal middle cerebral artery during labor. European Journal of Obstetrics \& Gynecology and Reproductive Biology, 109(1), 45-50.

14. Lakhkar, B. N., Rajagopal, K. V., \& Gourisankar, P. T. (2006). Doppler prediction of adverse perinatal outcome in PIH and IUGR. Indian Journal of Radiology and Imaging, 16(1), 109.

15. Long, P. A., \& Oats, J. N. (1987). Preeclampsia in Twin Pregnancy-Severity and Pathogenesis. Australian and New Zealand Journal of Obstetrics and Gynaecology, 27(1), 1-5.

16. Maged, A. M., Abdelhafez, A., Mostafa, W. A., \& Elsherbiny, W. (2014). Fetal middle cerebral and umbilical artery Doppler after 40 weeks gestational age. The Journal of Maternal-Fetal \& Neonatal Medicine, 27(18), 1880-1885.

17. Malik, R., \& Saxena, A. (2013). Role of colour Doppler indices in the diagnosis of intrauterine growth retardation in high-risk pregnancies. The Journal of Obstetrics and Gynecology of India, 63(1), 37-44.

18. Maulik, D. (1989). Basic principles of Doppler ultrasound as applied in obstetrics. Clinical obstetrics and gynecology, 32(4), 628-644.

19. Seal, S. L., Ghosh, D., Kamilya, G., Mukherji, J., Hazra, A., \& Garain, P. (2012). Does route of delivery affect maternal and perinatal outcome in women with eclampsia? A randomized controlled pilot study. American journal of obstetrics and gynecology, 206(6), 484-e1.

20. Vyas, S., Nicolaides, K. H., Bower, S., \& Campbell, S. (1990). Middle cerebral artery flow velocity waveforms in fetal hypoxaemia. BJOG: An International Journal of Obstetrics \& Gynaecology, 97(9), 797-803. 